\title{
Beam Test of the ATLAS Level-1 Calorimeter Trigger System
}

\author{
J. Garvey, S. Hillier, G. Mahout, T.H. Moye, R.J. Staley, J.P. Thomas, D.Typaldos, \\ P.M. Watkins, A. Watson, R. Achenbach, F. Föhlisch, C. Geweniger, P. Hanke, E-E. Kluge, \\ K. Mahboubi, K. Meier, P. Meshkov, F. Rühr, K. Schmitt, H.C. Schultz-Coulon, C. Ay, \\ B. Bauss, A. Belkin, S. Rieke, U. Schäfer, S. Tapprogge, T. Trefzger, G.A. Weber, \\ E. Eisenhandler, M. Landon, P. Apostologlou, B.M. Barnett, I.P. Brawn, A.O. Davis, \\ J. Edwards, C.N.P. Gee, A.R. Gillman A. Mirea, V.J.O. Perera, W. Qian, D.P.C. Sankey, \\ C. Bohm, S. Hellman, A. Hidvégi, S. Silverstein
}

\begin{abstract}
The Level-1 Calorimeter Trigger consists of a Preprocessor (PP), a Cluster Processor (CP), and a Jet/Energy-sum Processor (JEP). The $\mathrm{CP}$ and JEP receive digitised trigger-tower data from the Preprocessor and produce Regions-of-Interest (RoIs) and trigger multiplicities. The latter are sent in real time to the Central Trigger Processor (CTP) where the Level-1 decision is made. On receipt of a Level-1 Accept, Readout Driver Modules (RODs) provide intermediate results to the data acquisition (DAQ) system for monitoring and diagnostic purposes. RoI information is sent to the RoI builder (RoIB) to help reduce the amount of data required for the Level2 Trigger.
\end{abstract}

The Level-1 Calorimeter Trigger System at the test beam consisted of 1 Preprocessor module, 1 Cluster Processor Module, 1 Jet/Energy Module and 2 Common Merger Modules. Calorimeter energies were successfully handled throughout the chain and trigger objects sent to the CTP. Level-1 Accepts were successfully produced and used to drive the readout path. Online diagnostics were made using 4 RODs. Energy histograms were plotted and the integrity of data between the different modules

Manuscript received October 28, 2004.

J. Garvey, S. Hillier, G. Mahout, T.H. Moye, R.J. Staley, J.P. Thomas, D.Typaldos, P.M. Watkins and A. Watson are with the School of Physics and Astronomy, University of Birmingham, Birmingham B15 2TT, UK

R. Achenbach, F. Föhlisch, C. Geweniger, P. Hanke, E-E. Kluge, K. Mahboubi, K. Meier, P. Meshkov, F. Rühr, K. Schmitt and H.C. Schultz-Coulon are with the Kirchhoff-Institut für Physik, University of Heidelberg, D-69120 Heidelberg, Germany

C.Ay, B. Bauss, A. Belkin, S. Rieke, U. Schäfer, S. Tapprogge, T. Trefzger and G.A. Weber are with the Institut für Physik, Universität Mainz, D-55099 Mainz, Germany

E. Eisenhandler and M. Landon are with the Physics Department, Queen Mary, University of London, London E1 4NS, UK

P. Apostologlou, B.M. Barnett, I.P. Brawn, A.O. Davis, J. Edwards, C.N.P. Gee, A.R. Gillman, A. Mirea, V.J.O. Perera, W. Qian, and D.P.C. Sankey are with the Rutherford Appleton Laboratory, Chilton, Oxon OX11 0QX, UK

C. Bohm, S. Hellman, A. Hidvégi and S. Silverstein are with the University of Stockholm, Fysikum, SE-106 Stockholm, Sweden

(e-mail: gm@hep.ph.bham.ac.uk). was checked. All ATLAS detectors in the test beam were able to build full events based on triggers delivered by the Calorimeter Trigger System.

\section{INTRODUCTION}

At the full LHC design luminosity of $10^{34} \mathrm{~cm}^{-2} \mathrm{~s}^{-1}$, there will be approximately 23 proton-proton interactions per bunch crossing. The ATLAS Level-1 trigger is to reduce the $1 \mathrm{GHz}$ interaction rate to a trigger rate of $75 \mathrm{kHz}$ for input to the Level2 trigger. The reduction is performed by processing reducedgranularity data from the calorimeters and muon spectrometer. Potentially interesting events are selected by identifying electron/photon candidates, jets, single-hadron/tau candidates, missing transverse energy, and total transverse energy. Muon candidates are identified in a separate trigger. The Lever1 decision will be based upon a combination of these signals, and is made by the Central Trigger Processor (CTP). A complete description of the ATLAS trigger system can be found within this proceeding (NSS22-4).

A combined test beam has been run in autumn 2004 where samples of ATLAS subdetectors were tested. A liquid argon electromagnetic calorimeter and hadronic TileCal calorimeter were part of the test beam layout, and the Level-1 Calorimeter Trigger System took this opportunity to integrate our prototype boards. After a description of the trigger system down to the level of individual boards, this paper will report the results of this integration exercise which resulted in the generation of Level-1 Accepts based on data from both calorimeters.

\section{THE ATLAS LEVEL-1 CALORIMETER TRIGGER SYSTEM}

As shown as in Figure 1, the ATLAS Level-1 Calorimeter Trigger system consists of three subsystems, namely the Preprocessor (PP), electron/photon and tau/hadron Cluster Processor (CP), and Jet/Energy-sum Processor (JEP) [1]. The $\mathrm{CP}$ and JEP will receive digitized calorimeter trigger-tower data from the Preprocessor, and send trigger multiplicity information to the Central Trigger Processor via Common Merger Modules 


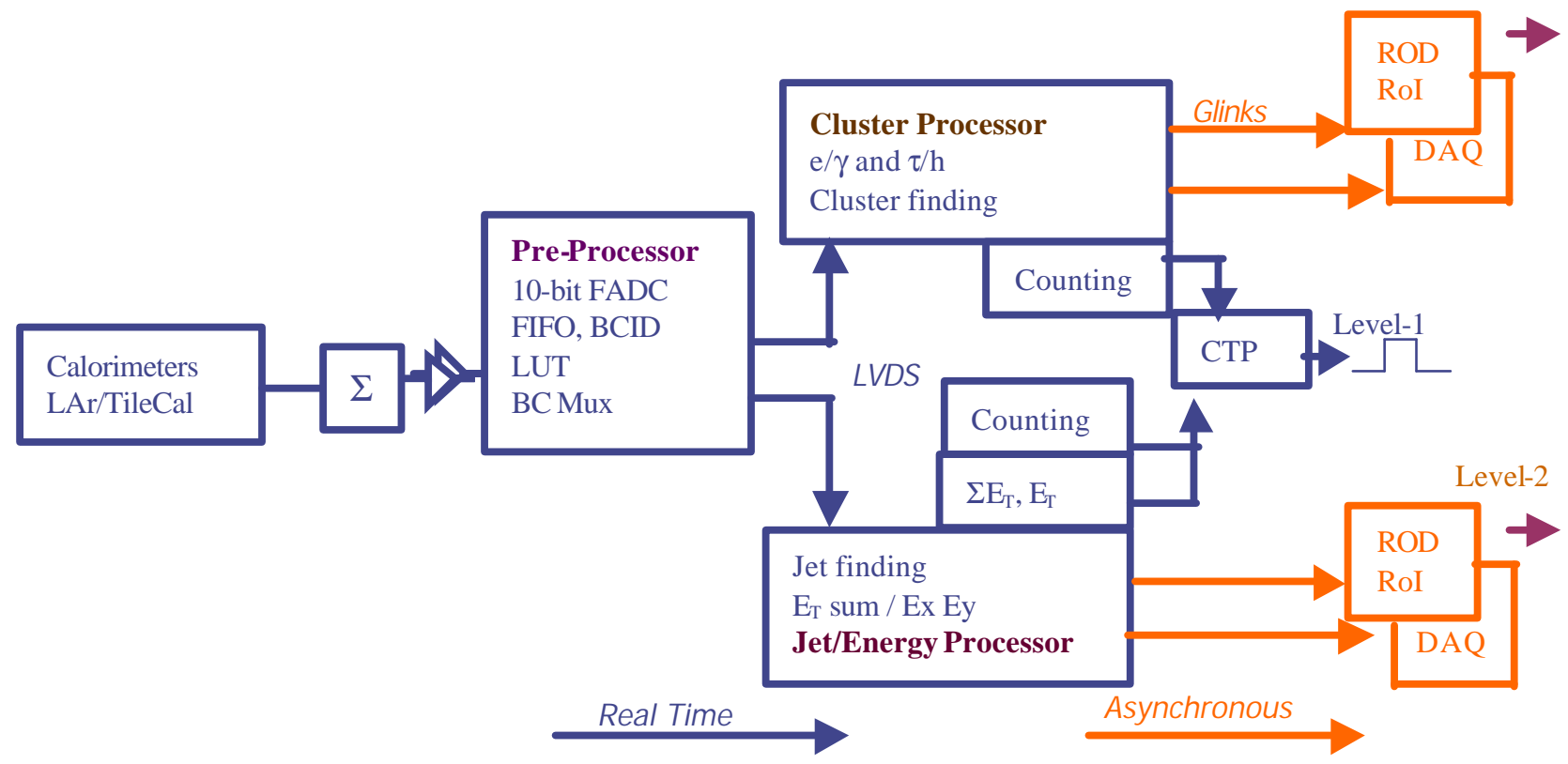

Figure 0 : The ATLAS Level-1 Calorimeter Trigger System layout

(CMM). Using Readout Driver modules (ROD), the CP and JEP will also provide regions-of-interest (RoI) information for the Level-2 trigger, and intermediate and final results to the data acquisition (DAQ) system for monitoring and diagnostic purposes.

\section{THE ATLAS LEVEL-1 CALORIMETER TRIGGER SYSTEM AT THE TEST BEAM}

During the test beam, both electromagnetic and hadronic calorimeters were available. On the calorimeter detectors, cells are combined to form trigger towers, with a reduced granularity of $0.1 \times 0.1$ in $\eta \times \phi$. The calorimeter trigger towers covered a total region of $0.2 \times 0.8$ in $\eta \times \phi$. A picture of the overall system during the test beam is shown in figure 2 .

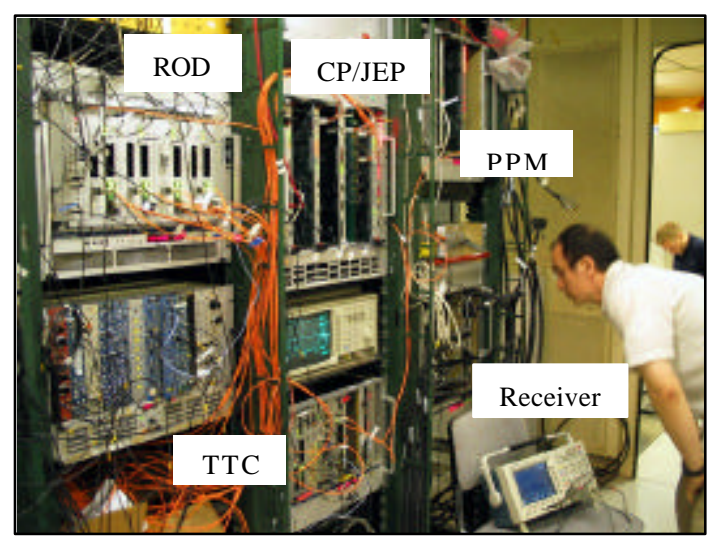

Figure 2 : The Trigger System at the Testbeam
A total of 5 crates were used:

- 1 Preprocessor crate containing 1 PPM

- 1 receiver crate

- 1 processor crate containing 2 CMMs, 1 JEM and 1 CPM

- 1 Control Crate for the clock control (TTCex) and local control of the Level-1 Accept rate

- 1 readout crate containing four RODs

The next sections describe the different modules used during the test beam, following the order of the data being processed through the system.

\section{A. The Preprocessor Module (PPM)}

The Preprocessor Module (PPM) digitises calorimeterpulses and prepares data for the CP and JEP. One PPM is shown on figure 3.

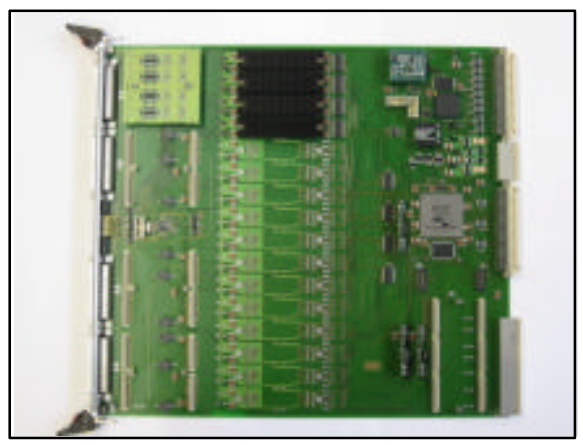

Figure 3 : The Preprocessor Module 
The board carries 16 Multi-Chip Modules (MCM) [2], each of them holding 4 12-bit FADCs, 1 ASIC and 1 timer chip (Phos4). Only half of them were used in the test beam (the picture shows only 4 MCMs mounted on the module) corresponding to a total of 32 channels. Analog calorimeter data are received from the front, and digital data are output to the CP and JEP systems using LVDS $400 \mathrm{MB} / \mathrm{s}$ serial links through the back of the board. Energy c alibration and bunch-crossing timing identification are performed on digital data, such as that shown on the figure 4.

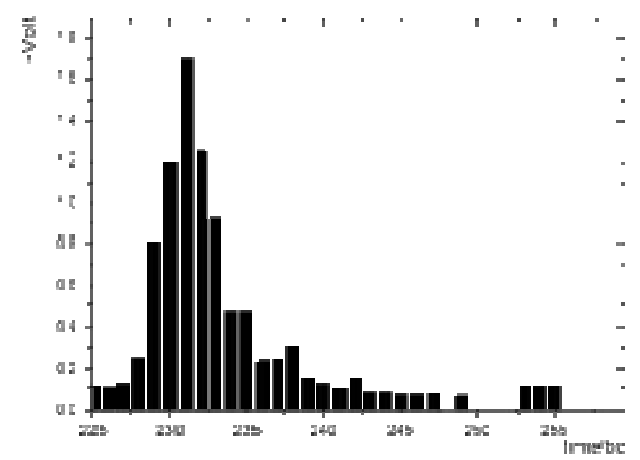

Figure 4 : A liquid argon pulse digitised by a PPM

\section{B. The Cluster Processor Module (CPM)}

The Cluster Processor Module (figure 5) was designed to find isolated electron/photon and hadron/tau clusters. The CPM received data from the PPM and sends its multiplicity results to the CMM, all through the backplane. On receipt of a Level-1 Accept, RoI and DAQ information are sent to the ROD over optical Glinks. The board carries a total of 32 FPGAs, the 8 largest providing the algorithm to find electron/photon and tau/hadron clusters. The algorithm used and its implementation in a FPGA could be found in [3].

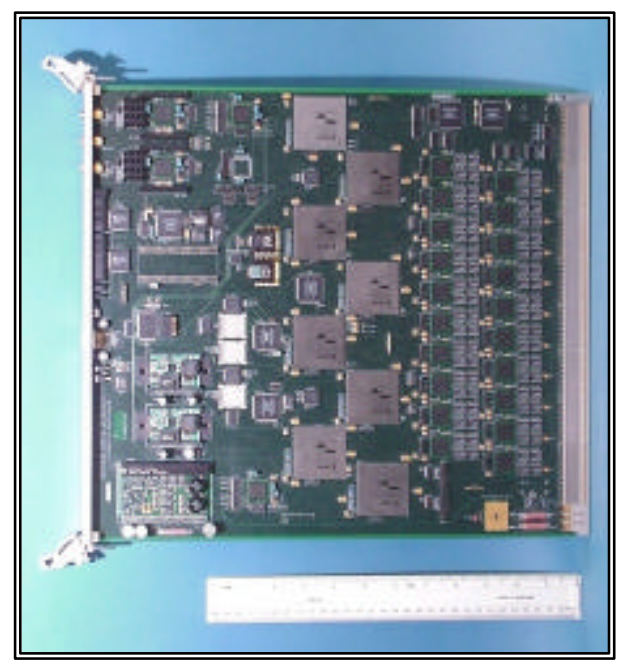

Figure 5 : The Cluster Processor Module

\section{The Jet/Energy Module (JEM)}

The Jet/Energy Module (JEM) [4] finds jets. It also performs the total energy summing and the pre-summings for the missing transverse energy. Like the CPM, the JEM receives its trigger data from the Preprocessor system using LVDS links, and sends jets multiplicities and energy sums to the CMM. It is a modular board where 4 input processor FPGAs do the summing of electromagnetic and hadronic elements to jet elements (figure 6).

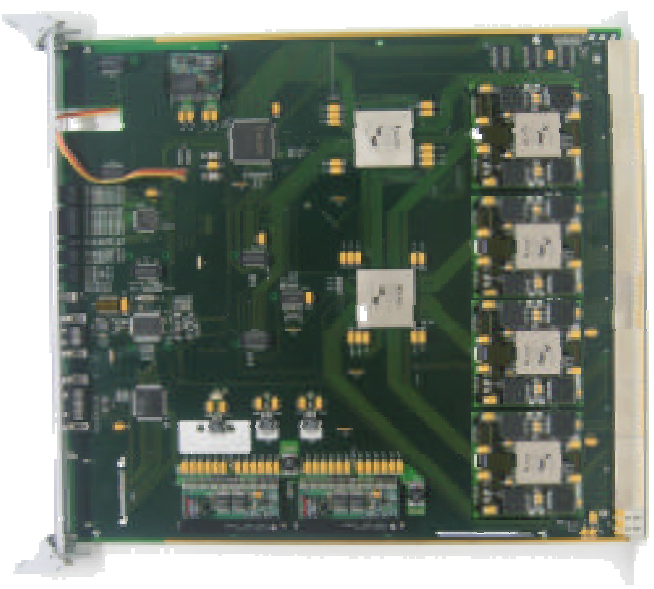

Figure 6 : The Jet/Energy Processor Module

\section{The Common Merger Module (CMM)}

The CMM (figure 7) gathers information from all CPMs or JEMs in a crate and sums it, then does system-wide sums and sends them to the CTP where the Level-1 decision is made [5].

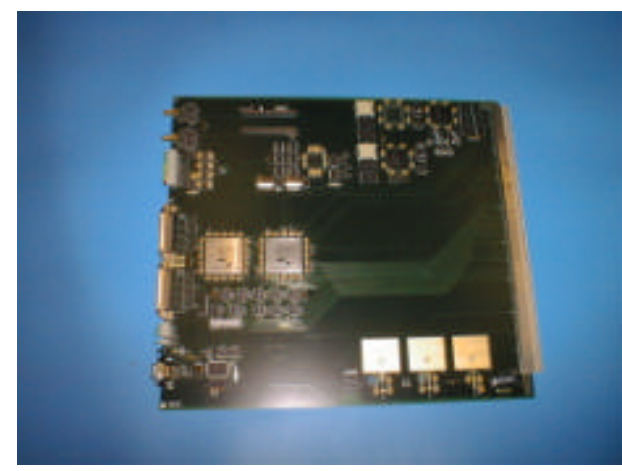

Figure 7 : The Common Merger Module

\section{E. The Readout Driver Module (ROD)}

The Readout Driver Module (ROD) [6] receives DAQ or RoI information generated by the trigger modules on each 
occurrence of Level-1 Accept. The ROD Module formats and prepares data to send it to the Readout Subsystem, from which full events are built.

\section{RESULTS}

During the test beam, the trigger system was successfully integrated with both liquid a rgon and Tile Calorimeters. The CMMs sent results to the CTP and thereby successfully produced triggers from the full trigger chain. The trigger menu was used to select electromagnetic cluster, jet and total energy triggers, and RoIs were successfully sent to the RoI Builder. The trigger system was run routinely under the main ATLAS Run Control system.

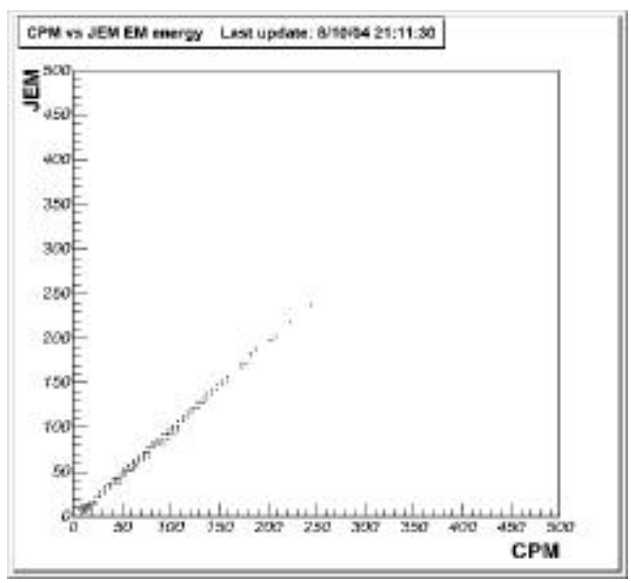

Figure 8 : The energy sent to the CPM versu the energy sent to the JEM.

The histogram above shows the clear correlation between energy sent to the JEM and the CPM during a run.

Figure 9 is the energy deposited in the hadronic calorimeter as a function of the energy recovered inside the CPM (courtesy of Pedro Amaral) of a proton run of $350 \mathrm{GeV}$. Energy calibration was not yet performed at this stage but there is a clear correlation between the hadronic calorimeter and the trigger system.

\section{CONCLUSION}

The integration of the Level-1 Calorimeter Trigger System at the test beam has been very successful. Trigger modules close to the final design have been shown to perform well. The generation of Level-1 Accepts based purely on information from the calorimeter has shown that the challenging Level-1 Calorimeter Trigger Sys tem is basically working.

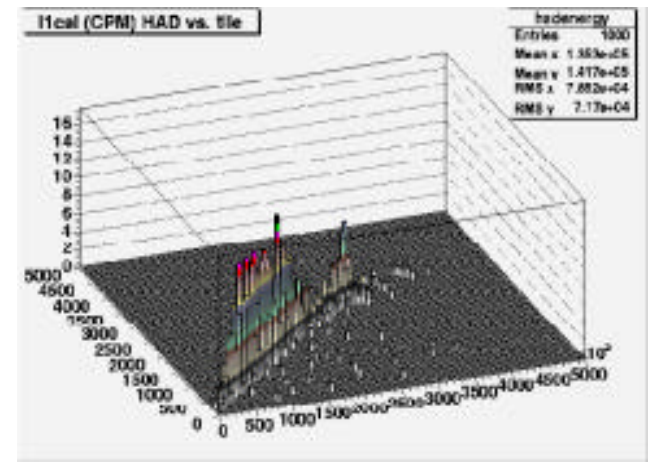

Figure 9 : Energy deposited inside the TileCal versus the energy received inside the $\mathrm{CPM}$

\section{REFERENCES}

[1] ATLAS First Level Trigger Technical Design Report, CERN/LHCC/98-14 and ATLAS TDR-12, 30 Jun 1998“

J.Garvey et al., "The ATLAS Level-1 Calorimeter Trigger Architecture", IEEE Trans. Nucl. Sci., Vol. 51, NO. 3, p356-360, June 2004

[2] "The Final Multi-Chip Module of the ATLAS Level-1 Calorimeter Trigger Preprocessor", $7^{\text {th }}$ Workshop on Electronics for LHC experiments, CERN/LHCC/2001-034, 22 October 2001,p.258

[3] "ATLAS Level-1 Calorimeter Trigger System: Subsystem Tests of a Prototype Cluster Processor Module", $9^{\text {th }}$ workshop on electronics for LHC experiments, CERN/LHCC-2003-055, $10^{\text {th }}$ November 2003, p.230

[4] J.Garvey et al., "ATLAS Level-1 Calorimeter Trigger System: Subsystem Tests of Jet/Energy Sum Processor Module", IEEE T rans. Nucl. Sci., Vol. 51, NO. 5, p2356-2361, Oct. 2004

[5] "One Size Fits All: Multiples Uses of Common modules in the ATLAS Level-1 Calorimeter Trigger", $7^{\text {th }}$ Workshop on Electronics for LHC experiments, CERN/LHCC/2001-034, 22 October 2001,p.253

[6] "Prototype Readout Module for the ATLAS Level-1 Calorimeter Trigger Processor", $7^{\text {th }}$ Workshop on Electronics for LHC experiments, CERN/LHCC/2001-034, 22 October 2001,p.263 